\title{
Téoros
}

Revue de recherche en tourisme

\section{Les événements au service de la ville ? Ou, la ville au service des événements!}

\section{André Langlois}

Volume 12, numéro 1, mars 1993

Québec, capitale et région touristiques

URI : https://id.erudit.org/iderudit/1078201ar

DOI : https://doi.org/10.7202/1078201ar

Aller au sommaire du numéro

Éditeur(s)

Université du Québec à Montréal

ISSN

0712-8657 (imprimé)

1923-2705 (numérique)

Découvrir la revue

Citer cet article

Langlois, A. (1993). Les événements au service de la ville ? Ou, la ville au service des événements! Téoros, 12(1), 36-39. https://doi.org/10.7202/1078201ar d'utilisation que vous pouvez consulter en ligne.

https://apropos.erudit.org/fr/usagers/politique-dutilisation/ 


\section{Les événements au service de la ville? Ou, la ville au service des événements!}

André Langlois*

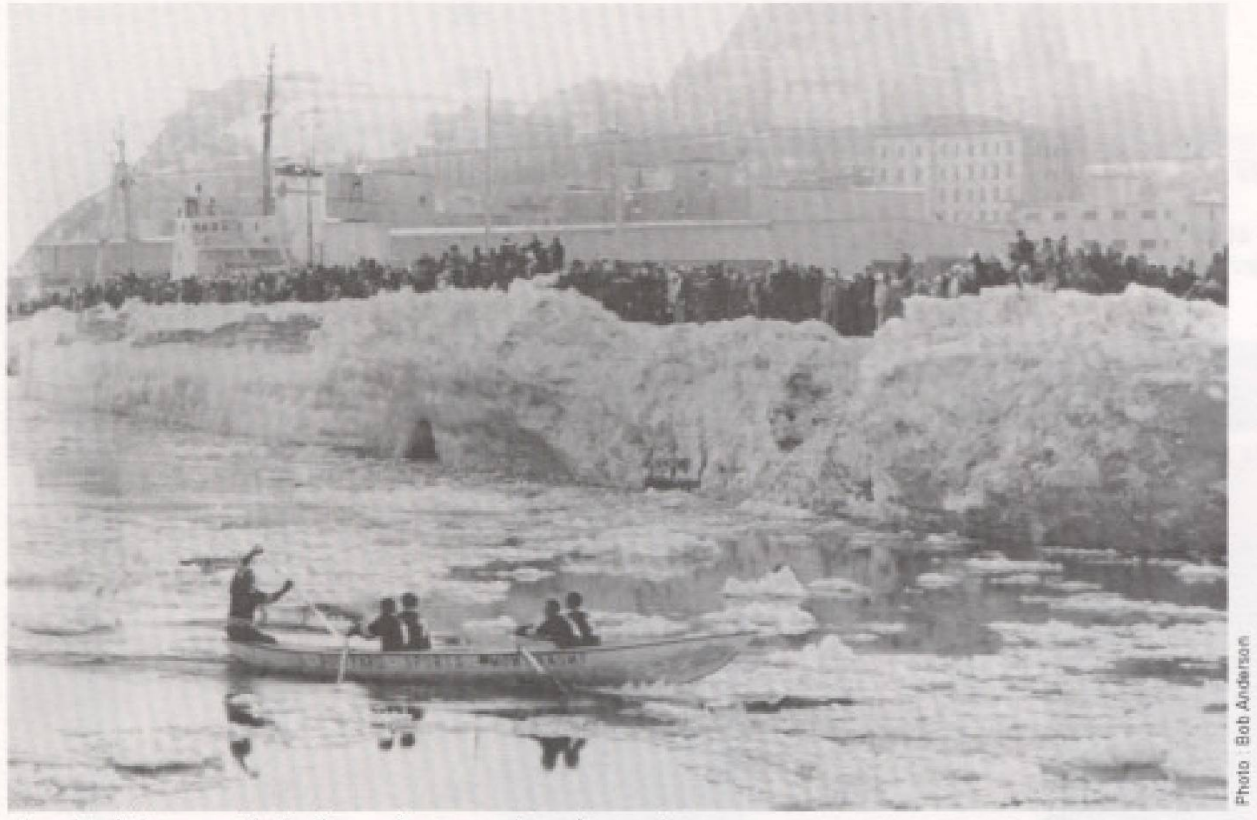

Certains evénements de Québec attireront toujours les touristes.

Le Bureau des arts et de la culture (BAC), créé en 1989 par la Ville de Québec, a pour premier mandat de doter cette ville d'une politique cohérente en matière d'événements et de projets spéciaux. Disposant d'un budget annuel de $3000000 \$$, le BAC effectue un travail remarquable dans un contexte aussi unique que difficile. Il collabore avec l'Orfice du tourisme et des congrès de la Communauté urbaine de Québec lorsque les événements ont un potentiel touristique.

Le troisième partenaire pour plusieurs événements est le Secrétariat à la Capitale, organisme qui n'a toujours pas reçu de budget adéquat de fonctionnement du gouvemement du Québec. Cette situation le relègue à un rồle qui reste discret. Pourtant, le secrétaire général associé au ministère du Conseil exécutif à titre de secrétaire associé à la Capitale, monsieur Jacques Demers, détenteur d'un doctorat en tourisme, possède une solide expérience des événements associés au développement touristique, expérience acquise au ministère du Tourisme où il était

Monsieur André Langlois est concepteur d'événements (il a formule le concept toriginal de Qubbec 1534-1984). sous-ministreassocié. MonsieurDemers a la volonté manifeste de doter la Capitale d'un véritable programme de communication par l'événement, orienté vers un tourisme culturel. De l'intention aux actions, il ne manque que la volonté politique du Conseil exécutif.

Montréal, c'est New York en plus petit; Québec, c'est Baie-Saint-Paul en plus gros! II ne faut donc pas s'offusquer que, pour les Montréalais, Québec soit Le Village. En matière d'événement, il faut bien admettre que la villeet la région de Québec ont toujours eu une approche locale. Le BAC et ses partenaires ont tout un defi à relever pour que la Capitale ait enfin une politique événementiellerationnelle. Onnepeutparler dela problématiquedesévénements àQuébec en ignorant sa situation politique et démographique ou en occultant Québec 84.

Au plan politique, Québec est la capitale du seul état francophone d'Amérique. Par la volonté des gouvemements issus du parti Libéral du Québec et du parti Québécois, cette capitale n'en a que le titre, car elle a perdu, au profit de la Métropole, les grandes institutions et les centres dedécision del'État. Une capitale que l'État refuse de reconnaître dans les faits; une capitale que l'État refuse de doter afin qu'elle puisse s'affirmer. Les autres pays d'Amérique du Nord, le Mexique, les Etats-Unis d'Amérique et même le Canada nous servent une admirable leçon en ayant une capitale qui soit autre chose qu'un prête-nom. En matière de grands événements dans la Capitale, le Secrétariat à la Capitale n'a pas pris jusqu'à présent ses responsabilités, pas plus qu'il n'estcapable de payer sa juste part.

Au plan démographique, les plus récents chiffres de Statistiques Canada ont établi la population de la région de Québec à 645 550 habitants dont 167517 ont résidence à Québec. De toute évidence, les 46 municipalités formant lagranderégionde Québec (rive Nord, rive Sud) ne contribuent pas au prorata de leur population au financement des grands événements. Les commanditaires dusecteur privévevaluent lapertinence de leur implication et le montant de commandite qu'ils y investiront en fonction du potentiel de spectateurs a l'événement et desretombéesmédiatiques, principalement desimages diffusées parla télévision. Avec un bassin de population six fois plus petit que Montréal, la commandite associable auxévénements à Québec se rétrécitcomme peau-de-chagrin. Difficulté supplémentaire: les décisions en matière de commandite se prennent à Montréal et non à Québec. Qu'en serait-il de la commandite dans la Métropole si ces décisions se prenaient a Toronto?

La situation de la télévision est encore plus dramatique. Selon sa politique de valorisation de l'unitécanadienne, le réseau national de Radio Canada accorde localement autant d"importance aux evénements se déroulant à Kamloops B.-C. ou à Terre-Neuve qu'à ceux de Québec. L'antenne locale de Radio Canada, quant a elle, accorde autant d'importance à Montmagny, à Rimouski et Gaspé qu'à la région de Québec. L'absence de Radio Canada n'est pas due à la volonté de ses artisans, tout au contraire; par exemple, c'està l'entêtementetà l'acharnementd'un réalisateur que l'on doit les images de la Transat Québec/Saint-Malo diffusées à la télévision Radio Canadienne. Le très 
montréalais réseau TVA concentre tous ses efforts et ses énergies à produire le moins possibleà Québecpendantque Radio Québec dépense ses déficits à Montréal. Reste la station locale de Quatre Saisons qui assure une remarquable couverture des activités et des événements de la région. C'est toutefois la Télévision communautaire, Télécom 9 qui assure la meilleure couverture dans le domainecultureletévénementiel. Laprestation de Télécom 9 est remarquablenon seulement au plan de la quantité maiségalement pour la qualité des reportages et des images.

Ce net désavantage de larégion de Québec au plan de la commandite et de la couverture médiatiqueseraitamoindri sile gouvernement du Québec adoptait vis-à-vis sa capitale un traitement comparable au traitement que le gouvernement du Canada, par le biais de la Commission delaCapitaleNationale, accorde à la région dºutawa.

L'autre réalité incontournable en matière d'événement dans la Capitale est le fantóme de Québec 84 qui habite toujours ses murs. Ce spectre est toujours vivant car l'échec de Québec 84 aura êté non pas le résultat des difficultés événementielles ou du déficit financier, mais de lhommerie. La bétise de l'homme a la vie longue. Il aura été plus facile au Québec d'oublier les scandales et le gouffre financier permanent et étemel du Stade Olympique de Montréal que la bêtise toute humaine des terrains de stationnements à Drummondville pour Québec 84, et pourtant...

Les événements sont très fragiles, surtout à leurs débuts, ce qui explique pourquoi, dans les années 1979,1980 et 1981, il aura suffi de quelques personnes pour pervertir cet evénement au potentiel exceptionnel. Richard Drouin, le capitaine appelé à la rescousse, après le naufrage annoncé de ce grand bâtiment touten voileset sans gouvernail, réussira tout de mêmeă livrer l'événement etă laisser en héritage à la ville de Québec la rénovation des secteurs du Vieux-Port el du VieuxQuébec en basse-ville.

Le fantôme exorcise, il faut, avant d'explorer des avenues pour une politique événementielle à venir, regarder objectivement, mais sans complaisance, la problématique des événements existants dans la Capitale. Commençons par un survol rapide de ses principaux événements: le Carnavald'hiver, le Festival d'été, la Transat Québec/SaintMalo, et le p'tit demier, Les Médiévales de Québec qui verra peut-être le jour en août
1993 si les commandites privées lui prêtent vie.

Le Camaval d'hiver de Québec a été le premierdes grandscarnavalsd' hiverd'aprèsguerre; son succès a été remarquable. Le Bonhomme Carnaval a cété - et demeure - un ambassadeur de marque pour tout le Québec. A sa trente-neuvième édition, le Camaval présente des symptômes d'essoufflement. Dans les années 1970 , les visiteurs remplissaient les hôtels durant les dix jours du Carnaval. Depuis, le Camaval a vécu ses heures sombres, celles du Carnaval de la bouteille; heureusement sa direction a su donner le coup de barre au bon moment et aujourd' hui on constate que le virage vers une fête pour la famille est réussi.

Les fêtes d'hiver se sont multipliées dans toutes les régions de neige du nord de l'Amerique. Notre Camaval a perdu son exclusivité, son monopole et n'attire plus les visiteurs comme dans les belles années. Le Camaval constitue cependant toujours un apport important et mesurable pour une industrie touristique qui en a bien besoin, surtout au mois de février.

Mais le Carnaval de Québec doit maintenant se partager les visiteurs, entre autres, avec Montreal et Ottawa. La Commission de la Capitale Nationale (CN) avait cré, en 1979 , l'événement dans cette dernière ville. $\mathrm{La}$ contribution financière de la CNN en 1993 a été ramenée de 1,2 à un million sur un budget de 2,5 millions; les commandites en biens et services sont de $800000 \$$. Les villes de Hull et Ottawa et la municipalité régionale d'Ottawa-Carleton (MROC) investissent la balance du budget, soit $700000 \$$. De quoi faire rêver le Carnaval de Québec qui reçoit $910000 \$(28 \%)$ en subventions sur un budget de $3250000 \mathrm{~s}$; la contribution du Québec s"élève à $325000 \$$.

L'organisation du Camaval n'est pas un cercle ferme; au contraire, une armbe de 2000 bénévoles y travaillent très fort. Un défí énorme les attend; comment plonger le Camaval et son Bonhomme dans la fontaine de jouvence sans les noyer, et les programmer pour l'an 2000 ?

Créé en 1967, le Festival d'été est l'enfant naturel de la Super Francofête. C est assurément la fêté la plus fréquentée par les gens. de la grande région de Québec. A sesdébuts, sous la direction de Noel Cormier, le Festival était principalement une fête de la découverte d'artistes venus de par le monde et la fête des artistes de la rue. Avec Danielle Bouchard à la direction générale, le Festival se dota d'un volet de musique classique. Avec la venue de deux directeurs généraux issus du monde du spectacle, messieurs Louis Coumoyer et Marcel Dallaire, le Festival a pris une orientation showbiz;les grands spectacles se multiplient, la présence d'une vedette ne suffit pas; qu'à cela ne tienne, on en alignera deux, trois et plus sur une même scène afin d'assurer le succès qui se mesure maintenant au nombre de spectateurs qui assistent au spectacle.

Doté en théorie d'une structure participative très démocratique, le Festival est cependant devenu, dans ses années showbiz, une société difficile d'acces avec une attitude très corporatiste. En 1993, une nouvelle équipe de direction est en place. Au fil des années, le Festival d'été s'est transformé en un excellentprogramme d'animation urbaine; mais peut-être au coût de son âme de festival. La raison d' etre du Festival de jazz de Montréal, du Festival de folklore de Drummondville et du Festival de l'accordéon de Montmagny sont l'évidence même; mais quelle est la vocation, la spécificité du Festival international d'été de Québec? Après 25 ans de créations, le Festival de Québec aura à se redéfinir, un processus normal, douloureux mais très sain.

Le Festival d'été pose la problématique de la gratuité des spectacles. La Villea-t-elle reçu de ses 165000 contribuables un mandat pour offrir des spectacles gratuits aux 645000 personnes de la grande région de Québec? La gratuite des spectacles rend très difficile la venueàQuébecàl'automne desspectacles payants. La Ville de Québec a-t-elle les moyens de faire les frais de spectacles gratuits? Ces argents ne seraient-ils pas mieux investis pour desévénements a l'automne ou au printemps?

Le concept de la Transat Québec/Saint-Malo était avant-gardiste en 1978 . Il précédait de quelques mois la grande premiere de la voilespectacle, la Route du Rhum orchestrée par le très parisien Michel Etevenon. La Route du Rhum, une Transat en solitaire a, pour réussir, plusieurs atouts: un départ en décembre dans la grisaille de Saint-Malo avec une arrivée dans un pays de soleil, la Guadeloupe. Les épreuves en solitaire ont toujours été la formule préférée des marins et des médias. Les marins sont bretons, les sponsors et les bateaux sont français. En 1984, les Transats en classe Open sur multicoques atteignaient leur apogée. De- 
puis c'est le déclin; aujourd"hui cette formule ne fait plus recette et il y a de moins en moins de ces Formule Un de la mer. De moins en moins de sponsors car la mode est aux défis, aux exploits tels le tour du monde en solitaire et sans assistance. Ce sont eux que suivent les médias. Pas de commandites sans médias, pas de médias sans spectacle, pas de spectacle sans argent, c'est la quadrature du cercle!

Voile Internationale de Québec, formée en août 1983, est l'organisateur de la Transat Québec/Saint-Malo. Cette société a but non lucratif estsous la présidence, ininterrompue depuis 1985, de Jean-Paul Massé. Cette corporation hermétique est très, très secrète. Depuis 1988, elle est entièrement financté par le secteur public, la commandite privée étant limitée à des prestations en biens et services. Le bilan événementielde la Transat 92 invite à la réflexion; pas de plan de commandite, pas de plan de communication. Au plan sportif, un seul bateau québécois sur la ligne de départ, le Cépal, un catamaran de course/croisière, cuvée 1988. Quelques marins québecois inscrits, souvent en tant que stagiaires payants sur des monocoques d'école-croisière. Desretombées médiatiques et touristiques quasi inexistantes dans nos marchés touristiques du Québec, du Canada et des États-Unis. Une épreuve sportive française au départ de Québec, payée par les Québécois... Un événement à caractère événementiel oui, mais la démonstration de sa rentabilitépourl'industrie touristiquereste à faire.

Menées avec brio et tambour battant, Les Médiévales de Québec sont prévues pour le mois d'août 1993; au coeur de la haute saison touristique... si les dollars des commandites du secteur privé sont au rendez-vous. Dommage que cette fête n'ait pas été planifiće pour se tenir au mois de septembre ou d'octobre, malgré toutes les difficultés imposćes par ces dates. Avec ses très belles images romantiques, Les Médiévales ont tout pour attirer le tourisme et séduire les commanditaires. Québeca-t-elle le besoin et les moyens de financer deux événements majeurs à l'été? La Ville s'est-elle posée cette question avant d'appuyer financièrement l'organisation des Médiévales et faire naître ainsi, auprès de ses créateurs passionnés, des espoirs de lendemains qui risquent d'être sans avenir?

C'est dans ce contexte très difficile que le Bureau des arts et de la culture travaille à la préparation d'une politique événementielle.
Cette equipe aura besoin du support et de l'appui de son patron, monsieur Jean-Paul L'Allier. Heureusement pour le BAC, monsieur L'Allier, un communicateur exceptionnel, est réceptif et sait évaluer à sa juste valeur unévénementexistant ouàcréer, pourvu qu'il soit bien informe et bien renseigné.

La Ville doit définir ses buts, ses objectifs et sa stratégie en matière événementielle. De ces définitions, naitra une grille objective d'évaluation des projets soumis. Le BAC, avec cette grille, deviendrait le guichet unique, la seule porte d'entrée par laquelle devraient obligatoirement et prioritairement passer tous les projets, sans exception.

La Ville devrait rendre cette grille accessible a tous. Bien informés, les auteurs de projets seraient en mesure de faire des propositions recevables par la Ville. Celle-ci devrait également avoir l'obligation de publier des appels d'offres, comme le font ses autres services, et d'inviter les auteurs et les promoteurs de projets à soumissionner pour réaliser les événements désirés.

A Québec, les auteurs et les promoteurs de projets événementiels résistent difficilement à la tentation de politiser la créativité en formant prématurément un Conseil d'administration pour des événements à l'état de projet. Ces conseils d'administration sont composés de personnalités très proches du pouvoir politique ou des organismes susceptibles de contribuer financièrement et d'introduire les projets auprès des élus. Pour éviter de politiser abusivement ce processus, il importe que la Ville considere le BAC comme tout autre service municipal et que ses procédures administratives ne soient pas différentes de celles des autres services administratifs.

La Ville pourrait adopter l'approche événementielle Vivaldi, la politique des Quatre saisons, quatre grands événements. Ces événements seraient de classe A et recevraient une assistance financière annuelle fixće sur base quinquennale. Le financement annuel decesquatre locomotives seraitassuré à partségales par le BAC, l'Officedu tourisme de la CUQ et le Secrétariat à la Capitale.

Les événements de classe $\mathrm{A}$ devraient obligatoirement:

- offrir un grand potentiel médiatique à l'extérieur de la région de Québec;
- valoriser le caractère culturel et politique de la capitale;

- générer, hors-saison touristique, des retombées économiques de visiteurs de l'extérieur de la région;

- être accessibleà l'ensemble de la population de la région.

Dans ces événements, la Ville devrait se comporter comme l'actionnaire majoritaire et l'hôte de la Fête, Le Bureau des arts et de la culture (et non les élus) devrait siéger d'office au comité exécutif et au comité de planification et de programmation de ces organisations. La Ville devrait également avoir un droit de vetodans ces organisations. C'est le prix à payer par les organisations qui souhaitent utiliser la Ville pour scène et les argents des contribuables pour payer ces fêtes.

La Ville ne devrait jamais s'engager dans un événement de classe A sans s'assurer d' avoir les ressources financières pour confier à des professionnels l'évaluation des foules, les retombées médiatiques et les retombées touristiques et économiques. Présentement, la Ville se fie quasi aveuglément aux prévisions et aux rapports fournis par les organisateurs et cette incurie fausse le débat. La Transat 92 illustre parfaitement les dangers de se fier aux rapports des promoteurs; dans une entrevue à la télévision, son directeur général évaluait les retombées touristiques à 8 millions puis, toujours dans le cours de la mềme entrevue, ce chiffre passeà 12 millions. Pour preuve de ces chiffres, il citait le fait que tous les vols entre Paris et Montréal étaient complets au mois d'août.

Voile Internationale de Québec affirmait, très sérieusement, qu'au départ de la Transat 92 , il y avait 350000 spectateurs, affirmation reprise par Le Soleil. Or, un relevé photographique exhaustif et systématique de la foule, faità laterrasse Dufferin, permet plutôt de parler d'une foule pouvant varier entre 25 000 et 50000 spectateurs, ce qui est d'ailleurs fort honorable. Là où le bâtblesse, c'est que Voile Internationale utilisera ces chiffres et statistiques gonflés pour justifier ses demandes de subvention, coupures du Soleil à l'appui. La région de Montréal utilise maintenant des experts indépendants pour ce travail, à la demande même des commanditaires; leurs résultats confirment que les évaluations de foule sont généralement surévaluées de 5 à 10 fois. Même avec la meilleure des bonnes volontés, il est impos- 
sible d'estimer à l'oeil les foules de plus de mille personnes, surtout lorsqu"elles sont réparties sur un grand territoire.

Dans le climatéconomique actuel, les événementsdoivent réduire leurs frais d'opération. Puisque les quatre événements proposés se dérouleraient à des périodes annuelles differentes, la Ville devraitprendrele leadership et exiger que ces quatre événements se regroupent sous un seul toit et partagent des services, des equipements et du personnel. Cette solution ne viendra assurément pas de ces organisations. A la demande de la Ville, ce regroupement pourrait agir en tant que structure d'accueil et d'incubateur pour les futurs projets. Les économies générées par cette rationalisation des effectifs seraient très grandes; elles pouraient permettre à ces organisations de se doter collectivement d'equipements a la fine pointe de la technologie, ce qu'elles ne peuvent se payer seules. Ces économies pourraient également rentabiliser l'engagement des meilleurs spécialistes de la communication événementielle au service de tous.

La Ville devrait inciter les événements existants et obliger les futurs projets Evénementiels à adopter la politique d'inclure d'office à leur budget d'opération un dollar en communication par dollar investi dans l'organisation. Après tout, il s'agit bien de communication événementielle. Cette politique réussit fort bien en France où elle est la règle et non l'exception. Lorsqu'un événement prétend avoir un impact à l"extérieur sur le tourisme, il se doit d'assurer sa propre communication dans les médias spécialises. L'Office du tourisme a toute la compétence, le réseau et le personnel pour publiciser et distribuer le produit et ses publications auprès des intervenants touristiques.

Un tel regroupement de services permettrait enfin à ces organismes d'assurer la captation des spectacles de ces événements et de produire et mettre en marché auprès des diffu- seurs leurs propres émissions; solution fort rentable qui a fait ses preuves ailleurs.

Le Bureau des arts et de la culture devrait inclure dans sa politique une classification des êvénements, comme le fait le Bureau international des expositions. Des critères tels I'incidence touristique, culturelle, sportiveet la récurrence de ces projets serviraient a cette classification. Une seconde classe d'événements de classe B pourrait inclure les événements devant relever uniquement de la Ville. L'unique porte d'entréce serait le BAC et la grille analytique serait le premier test pour les auteurs et les promoteurs de ces projets. Les evénements retenus devraient faire l'objet d'une convention avec la Ville prévoyant un désengagement progressif des subventions de la Ville sur trois ans.

Les événements doivent être au service des collectivités et non l'inverse et s'ils prétendent générer des retombées pour le tourisme, ils devront travailler en aval eten amont avec cette industrie.

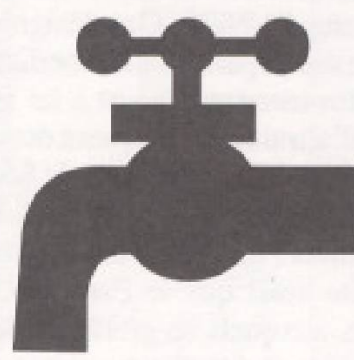

\section{Le Centre de Services Environnement Jeunesse (CSEJ)}

met à la disposition des animateurs et animatrices oeuvrant auprès des jeunes de 15 à 30 ans une trousse de vulgarisation scientifique consacrée à la thématique de l'eau.

\section{Vivre l'eau}

offre par la diversité de ses composantes, de multiples possibilités d'utilisation.

Document de vulgarisation scientifique, cahier pédagogique, vidéo, exposition d'affiches contribuent à une meilleure compréhension de la problématique et des enjeux actuels.

La trousse de vulgarisation scientifiqueVivre l'eau sera disponible dès juin 1993.

Réservez dès maintenant votre exemplaire en appelant à frais virés au: (514) $252-3016$ 